\title{
Gemeindepsychiatrie im 21. Jahrhundert
}

\author{
Community Psychiatry in the $21^{\text {st }}$ Century
}

Autor

Institut
Martin Hambrecht

Klinik für Psychiatrie, Psychosomatik und Psychotherapie, Evang. Krankenhaus Elisabethenstift, Darmstadt

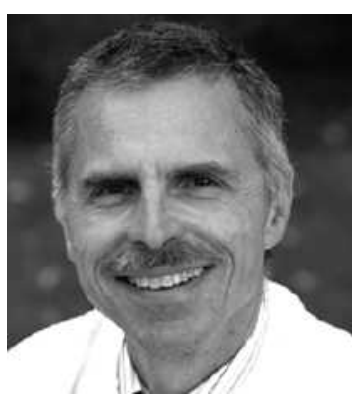

Prof. Dr. med. Dr. phil. Martin Hambrecht

\section{Bibliografie}

DOI 10.1055/s-2007-970938

Psychiat Prax 2007; 34:

e13-e16

(c) Georg Thieme Verlag KG

Stuttgart · New York .

ISSN 0303-4259

Korrespondenzadresse

Prof. Dr. med. Dr. phil.

Martin Hambrecht

Klinik für Psychiatrie, Psychosomatik und Psychotherapie, Evang. Krankenhaus Elisabethenstift $\mathrm{gGmbH}$ Landgraf-Georg-Straße 100 64287 Darmstadt

hambrecht.martin@eke-da.de
Auch wenn an vielen Orten lokale „gemeindepsychiatrische“ Initiativen engagierter Bürger, Landesherren oder Kirchen bis ins 18. und 19. Jahrhundert zurückweisen, formierte sich das Konzept der Gemeindepsychiatrie im deutschen Sprachraum erst in Verbindung mit der Psychiatrieenquete 1975. Insbesondere in den angelsächsischen Ländern hatte sich der Begriff „Community psychiatry“ bereits 20 Jahre früher fest etabliert.

Community psychiatry bedeutete dabei „Psychiatry focusing on detection, prevention, early treatment, and rehabilitation of emotional and behavioral disorders as they develop in a community“ [1]. Damit wird Community psychiatry durch ihre Inhalte definiert. Deutsche Definitionen stellten hingegen eher Strukturen in den Mittelpunkt und kennzeichneten beispielsweise noch 1999 „gemeindenahe Psychiatrie“ als „seit 1964 praktizierte Organisationsform psychiatrischer Behandlung. Die Leistungen psychiatrischer Krankenhäuser, niedergelassener Ärzte, von Übergangseinrichtungen, Gesundheitsbehörden und Beratungsstellen werden organisatorisch zusammengefasst und systematisiert" [2]. Ein Vergleich der beiden Definitionen führt zur These, dass es der deutschen Psychiatrielandschaft an inhaltlichen Visionen mangelt und Organisationsfragen zu große Aufmerksamkeit geschenkt wird.

In der Psychiatrieeenquete von 1975 wurden zentrale Forderungen formuliert, die unter anderem durch mehr Gemeindepsychiatrie umgesetzt werden sollten: Gleichstellung psychisch mit körperlich Kranken; Gemeindenähe; Kontinuität in der Behandlungskette und Schaffung von Übergangseinrichtungen und Verkleinerung der Großkrankenhäuser, Schaffung kleinerer Behandlungseinheiten.

Obwohl die Gemeindepsychiatrie der letzten 30 Jahre nach besten Kräften für die Realisierung dieser Ziele arbeitete und dabei immer wieder sehr erfolgreich war, bleiben diese Forderungen der Enquete auch heute aktuell. Die Gleichstellung der psychisch mit den körperlich Kranken ist auf dem Papier vollzogen. Im Großen wie im Kleinen wird aber jeden Tag deutlich, dass hier für alle Beteiligten noch viel zu tun bleibt. Ein gravierendes Beispiel ist die Unterfinanzierung der ambulanten nervenärztlichen Versorgung. Kleine Beispiele erleben wir immer wieder, wenn z.B. psychisch Kranke ablehnend behandelt werden, wenn sie körperlich erkranken.

Die Gemeindenähe ist vor allem in den Flächenländern noch nicht vollständig realisiert [3]. Wo die alten Landeskrankenhäuser noch dominieren, gibt es auch heute Anfahrtswege von über $50 \mathrm{~km}$. Gemeindenähe ist allerdings nicht nur in Kilometern zu messen. Isoliert und damit gemeindefern können psychisch Kranke, Sucht- oder Alterskranke auch in Gettos mitten in der Stadt leben, wenn sie keine Möglichkeit haben, am Gemeindeleben teilzuhaben. Kontinuität in der Behandlungskette bleibt eine ebenso schwierig zu realisierende Aufgabe angesichts der Zersplitterung der Kostenträgerlandschaft, der Aufspaltung von Budgets und der vielerorts unübersichtlichen Vielzahl von Diensten.

Auch wenn die Psychiatriereform hinsichtlich des Versorgungssystems nicht als abgeschlossen gelten kann [3], wurden die strukturellen Forderungen der Psychiatrieenquete noch am besten umgesetzt. Häuser sind leichter zu bauen als Herzen umzustimmen. Übergangseinrichtungen (Tageskliniken, betreute Wohn- und Arbeitsformen) wurden in den Ballungsgebieten geschaffen, fehlen aber in vielen ländlichen Regionen. Teilweise durch Umsetzung in psychiatrische Heime, teilweise aber auch durch Abtretung von Pflichtversorgungsgebieten an Abteilungspsychiatrien wurden die ehemaligen psychiatrischen Großkrankenhäuser deutlich verkleinert. Heute verfügen die 202 Fachkrankenhäuser für Psychiatrie und Psychotherapie im Mittel über 144 Betten. 
Die 220 Abteilungen an Allgemeinkrankenhäusern haben im Mittel 81 Betten. Insgesamt gibt es über 8000 Tagesklinikplätze, sodass knapp $20 \%$ aller Klinikplätze in Tageskliniken vorgehalten werden.

\section{Gemeindepsychiatrie heute \\ $\nabla$}

Wenn wir Gemeindepsychiatrie als angewandte Sozialpsychiatrie verstehen, dann wird deutlich, warum die Diskussion um die Gemeindepsychiatrie immer eine politische Debatte war und ist. Diese Debatte hat sich in den zurückliegenden 30 Jahren deutlich gewandelt. War es damals allgemein akzeptiert, dass Sozialpsychiatrie auch gesellschaftspolitische Ziele verfolgt, so müssen wir in den zurückliegenden Jahren eine Entpolitisierung der Diskussion feststellen. Wurde in den 60er- und 70er-Jahren noch explizit der Abbau von Ungleichheiten, gelebte Solidarität und soziale Gerechtigkeit eingefordert, so stehen heute Verwaltungsbegriffe wie „Zielgruppenmanagement“ oder „Ressourcenallokation“ im Mittelpunkt.

Was aber ist nun zeitgemäß in der Gemeindepsychiatrie? - Ist man schon modern, wenn man managt und nicht mehr verwaltet? Ist man modern, wenn man personenzentriert umfangreiche Rehabilitationspläne zu Papier bringt? [3] In die Mottenkiste gehören zweifellos Begriffe wie „extramurale Dienste“ oder auch „komplementäre Dienste“, die die Sichtweise einer überkommenen auf Großkrankenhäuser zentrierten, in Anstaltsmauern eingefriedeten Psychiatrie illustrieren. Heute verbringt der chronisch psychisch Kranke (hoffentlich) den allergrößten Teil seines Lebens außerhalb des Krankenhauses, sodass in einer modernen Gemeindepsychiatrie allenfalls das Krankenhaus als „komplementär“ zu bezeichnen ist. Der stationäre Bereich kann vor dem Hintergrund erzwungenermaßen sinkender Verweildauern meist nur die Funktion der Krisenintervention übernehmen. Die eigentliche Versorgung hingegen geschieht im außerstationären Bereich.

Gemeindepsychiatrie beinhaltet immer auch die Forderung, dass sich Kommunen mitverantwortlich für die psychiatrische Versorgung im Sinne der Daseinsfürsorge für ihre Bürger mit ihren Grundbedürfnissen fühlen. Hier wurde in den zurückliegenden Jahrzehnten viel erreicht. Angesichts der zunehmenden Zersplitterung der Dienste werden Aufgaben der Vernetzung und Koordination immer wichtiger. Da Kommunen im Idealfall keine eigenen ökonomischen Interessen haben, sollten diese Aufgaben auch bei den Kommunen verbleiben. Daneben spielen Kommunen für chronisch psychisch Kranke eine wichtige Rolle in der Daseinsfürsorge durch die Gewährung von Transferleistungen, aber auch bei der immer wichtigeren Aufgabe der Wohnungsversorgung für psychisch kranke Wohnungslose, deren Hilfebedarf und Anzahl zunehmende Probleme stellt.

Die Rolle der einzelnen Akteure innerhalb der Gemeindepsychiatrie hat sich in den letzten Jahren gewandelt und wird sich weiter wandeln. Aufgrund der gesundheitspolitisch gewollten Strangulation der niedergelassenen Psychiater wird es diesen auch bei größtem Einsatz kaum noch möglich sein, eine echte Rolle in der sozialpsychiatrischen Versorgung zu spielen. Auf die Dauer kann sich kein niedergelassener Kollege den unbezahlten Extraeinsatz erlauben, der hier notwendig wäre. Niedergelassene Psychiater und Psychotherapeuten werden sich aber weiterhin um die mündigen, kooperativen, d. h. letztlich weniger schwer Kranken kümmern. Komplexe, nur multidisziplinär zu versorgende Krankheiten und Behinderungen werden hingegen zunehmend in den Ambulanzen der Krankenhäuser oder arbeitsteilig durch verschiedene Dienste geleistet werden.

Dabei wird die Versorgungssituation für chronisch Kranke immer komplexer und auch für die Profis oft nicht mehr zu überblicken. Allein innerhalb der Kommune sind schon bis zu vier Dienststellen mit einem einzigen Patienten befasst: Betreuungsstelle, Sozialpsychiatrischer Dienst, Sozialamt, Wohnungsamt. Dazu kommt dann die behandelnde Arztpraxis oder Klinikambulanz, der gesetzliche Betreuer, der Träger für das betreute Wohnen, evtl. eine Rehabilitationswerkstatt bzw. die Agentur für Arbeit, möglicherweise ein Psychotherapeut und vorübergehend die Hilfeplankonferenz, eine Krankenhausstation oder Tagesklinik. Der Abstimmungsbedarf steigt. Immer mehr Sitzungen werden erforderlich. Wir sprechen mehr über den Patienten als mit dem Patienten. Die typische Antwort des Versorgungssystems: mehr Bürokratie, mehr Pläne, mehr Formalismus. Nötig ist eine radikale Vereinfachung: trägerübergreifende Zusammenfassung von Dienststellen und weitreichende Kompetenzen für die Hauptbezugsperson, den „Case manager“.

Mit dem politisch gewollten Bedeutungsverlust der niedergelassenen Ärzteschaft stellt sich zunehmend die Frage nach der Versorgung am Abend, in der Nacht und am Wochenende. Die Mitarbeiter in der psychiatrischen Versorgung haben hier unterschiedliche Bedürfnisse und zeigen durchaus eine unterschiedliche Mentalität in Abhängigkeit von der Institution, in der sie arbeiten. Das „Dienstzeitdenken“ ist in den unterschiedlichen Kulturen Krankenhaus, Praxis und öffentlicher Verwaltung sehr unterschiedlich ausgeprägt, was zweifellos in der zunehmend notwendigen vernetzten Versorgung einige Anpassungsleistungen erfordern wird.

Separat bezahlte psychiatrische Notdienste bieten hier die Möglichkeit das Dienstzeitdenken zu überwinden, benötigen jedoch eine eigene Finanzierung, die angesichts der knappen öffentlichen Kassen im Wesentlichen nur über Spenden und freiwillige Zuwendungen möglich ist. Letztlich ist eine echte Deinstitutionalisierung aber auch 30 Jahre nach der Psychiatrieenquete nur möglich, wenn sie mit einer entsprechenden Flexibilisierung der Arbeitszeiten der Mitarbeiter für eine ambulante Versorgung rund um die Uhr einhergeht.

\section{Bedarfsbezogener Mitteleinsatz \\ $\nabla$}

Hier schließt sich eine weitere Forderung einer modernen Gemeindepsychiatrie an: Der personenzentrierte Ansatz [4] kann nicht nur von denen erwartet werden, die behandeln, beraten, begleiten oder beim Wohnen und in der Freizeit betreuen, sondern muss auch von denjenigen wahrgenommen werden, die diese Leistungen finanzieren. Moderne Gemeindepsychiatrie heißt Koordination - Koordination aber nicht nur aufseiten der Leistungsanbieter, sondern auch aufseiten der Kostenträger.

Realität ist heute eine extreme Zersplitterung der Kostenträger, deren nachvollziehbares Eigeninteresse darin besteht, möglichst nachzuweisen, dass man nicht zuständig ist. So beschäftigen einzelne Krankenkassen eigens Berater mit dem Ziel, Leistungen in den Bereich der Rehabilitation zu verschieben, weil diese von den Rentenversicherern zu finanzieren ist. Umgekehrt verschleppen Kostenträger Entscheidungen über geschützte Wohnformen, sodass Krankenkassen für Patienten ohne Wohnperspektive weiter im Krankenhaus aufkommen müssen. Alltag sind auch die zahlreichen Konflikte zwischen örtlichen und überörtlichen Sozialhilfeträgern über die Zuständigkeit bei der 
Versorgung psychisch kranker Wohnungsloser. Hier könnten viele Stunden Sozialarbeit nützlicher eingesetzt werden als in ermüdender Korrespondenz mit den verschiedensten Institutionen. Gemeindepsychiatrie muss deshalb fordern, dass die Kostenträger sich an einen Tisch setzen und hier ebenso personenzentriert zusammenarbeiten, wie dies von den Leistungsanbietern mit Recht gefordert und realisiert wird.

Bestand vor 30 Jahren ein enormer Nachholbedarf auf nahezu allen Gebieten der Versorgung psychisch Kranker, so geht es heute um eine faire Verteilung der zur Verfügung stehenden Ressourcen. Wie kann dies gelingen? Epidemiologische Daten können dazu beitragen, den Hilfebedarf abzuschätzen. Was brauchen 300000 durch Schizophrenie chronisch behinderte Menschen, 2,5 Mio Alkoholkranke, 8 Mio depressive Menschen und über 1 Mio Demenzkranke? Nicht nur die Häufigkeit, sondern auch das Ausmaß der Krankheitsschwere und der resultierenden dauerhaften sozialen Behinderung sind zu berücksichtigen.

Vor dem Hintergrund der zentralen Forderung der Psychiatrieenquete nach Enthospitalisierung und gemeindenaher Versorgung chronisch Kranker war das gemeindepsychiatrische Hilfesystem traditionell vor allem auf schizophrene Psychosen hin ausgerichtet. Suchterkrankungen werden in einem separaten System versorgt und die in den letzten Jahren zunehmend in den Mittelpunkt der Aufmerksamkeit gerückten Demenzerkrankungen wiederum in einem anderen Hilfesystem. Diese Hilfesysteme unterscheiden sich nicht nur in ihrem Personal und therapeutischen Institutionen, sondern auch in den jeweils zuständigen Verwaltungen und deren spezielle gesetzlichen Regelungen und Verwaltungsvorschriften, z. B. für Fördermittel.

Die Probleme, die aus diesen abgetrennten Hilfesystemen resultieren, werden zunehmend deutlich. Wir beobachten in den letzten Jahren eine Verschiebung des Diagnosespektrums, so dass die Zuordnung zu den drei Hauptgruppen (Psychose, Sucht, Gerontopsychiatrie) immer schwieriger wird. Insbesondere bei der Doppeldiagnose von Psychose und Sucht, bei den Persönlichkeitsstörungen und bei den alt werdenden psychisch Kranken wächst der Bedarf. Es entsteht die zwingende Notwendigkeit zur Zusammenarbeit zwischen den bislang separierten Hilfesystemen. Die Beharrungskräfte innerhalb der jeweiligen Systeme haben sich hierauf noch nicht ausreichend eingestellt. Die Fördermittel für Jugendhilfe, Altenhilfe, Sozialpsychiatrie oder auch Psychosomatik werden noch immer separat verwaltet. Auch vonseiten der Kostenträger besteht der Wunsch nach Trennung, um die Zuständigkeiten besser abgrenzen zu können. Dies wird jedoch den Bedürfnissen der Betroffenen immer weniger gerecht.

Die Grundbedürfnisse sind zwar relativ ähnlich: Wohnen, Beschäftigung, Freizeitgestaltung, medizinische und psychologische Therapie werden von allen Diagnosegruppen nachgefragt. Aufgrund der unterschiedlichen Defizite und Verhaltensauffälligkeiten werden jedoch jeweils besondere Erfahrungen von den Betreuungspersonen verlangt, die sich angesichts der wachsenden Zahl von Doppel- und Dreifachdiagnosen komplexeren Aufgaben gegenüber sehen. Die Anforderungen an die Flexibilität der Mitarbeiter und an ihre Qualifikation steigen demnach. Hier hat die Allgemeinpsychiatrie, zumal als Abteilung am Allgemeinkrankenhaus, einen Erfahrungsvorsprung, weil sie sich traditionell mit allen Diagnosegruppen und mit komplexen Problemstellungen auseinanderzusetzen hat. Im Bereich der ambulanten bzw. gemeindepsychiatrischen Versorgung besteht hingegen Nachholbedarf.

\section{Stigma und Ghettobildung}

Gemeindepsychiatrie arbeitet im selben Umfeld, in dem auch soziales Stigma erlebt und erlitten wird. Moderne Gemeindepsychiatrie muss sich deshalb mit Ausgrenzung, Abwertung, Entmenschlichung psychisch Kranker auseinandersetzen. Stigmatisierung umfasst aber nicht nur ausgrenzende Verhaltensweisen, sondern auch die damit einhergehenden Gedanken und Gefühle: Vorurteile, Ängste, Aggressionen gegenüber dem Fremden, dem Anderen, dem Unvorhersehbaren - C. G. Jung hätte gesagt, „dem Schatten“ in uns allen.

Stigmatisierung hat also eine psychologische Funktion für den Stigmatisierer, und das ist einer der Gründe, weshalb jahrelange engagierte Öffentlichkeitsarbeit es nicht vermocht hat, die Stigmatisierung psychisch Kranker zu beenden. Bei großen Umfragen denken beim Stichwort „Schizophrenie“ viele Befragte an Gefahr und Gewalt, aber nur wenige an Behandlung. Die Bevölkerung ist zwar besser informiert, die soziale Distanz zu psychischen Kranken hat aber eher noch zugenommen. Allgemeine Aufklärung ist zwecklos. Da macht eine einzige Schlagzeile die bescheidenen Fortschritte mühsamer Kampagnen im Handumdrehen zunichte. Aber lokale Aktionen mit direkter Begegnung zum Beispiel von Schülern mit psychisch Kranken [5] und der Möglichkeit zu emotionalem Lernen ist sinnvoll.

Wie reagiert ein Mensch, der Stigmatisierung erlebt? Diese „zweite Krankheit“, wie Asmus Finzen [6] das Stigma nannte, resultiert in Hoffnungs- und Hilflosigkeit und in Depression. Aus Selbstschutz kommt es verständlicherweise zum Rückzug und zum Zusammenschluss mit ebenfalls Betroffenen. Die Interessen der gesunden Mehrheit und der betroffenen Minderheit führen zu einer Ghettoisierung chronisch psychisch Kranker, die wir also gemeindepsychiatrische Subkultur heute in allen Städten vorfinden. Moderne Gemeindepsychiatrie muss als Realität akzeptieren, dass viele chronisch Kranke sich ein Netz von Betreuungspersonen und Therapeuten als Ersatz für ein „normales soziales Netz" schaffen und im Übrigen fast nur Kontakt zu anderen Betroffenen pflegen. Viele erleben nur noch hier Verständnis und Akzeptanz anstelle von Ablehnung und Stigmatisierung.

Vor diesem Hintergrund sind Integrationsangebote zwar nötig, forcierte Integration trägt hingegen das Risiko einer Dekompensation in sich. In diesem Kontext steht die aktuelle Diskussion [7], die sich aus der Etablierung spezieller Angebote für chronisch psychisch Kranke, z.B. im Bereich von Arbeiten und Wohnen, ergeben hat. Zunehmend wird infrage gestellt, dass separate Werkstätten für Rehabilitation oder Wohnheime für psychisch Kranke geschaffen und erhalten werden. Vielmehr wird von verschiedenen Autoren gefordert, stärker die allgemeinen Angebote innerhalb einer Kommune in Anspruch zu nehmen und z.B. betreute Arbeitsplätze in ganz normalen Firmen oder für die Freizeit Integrationsangebote in normalen Sportvereinen zu schaffen, statt eigene Sportgruppen für psychisch Kranke ins Leben zu rufen. Nur so bestehe eine Chance zur Integration.

Moderne Gemeindepsychiatrie bietet jedoch ein breites Angebot, damit je nach sozialer Kompetenz dem psychisch Kranken die Möglichkeit gegeben ist, sich entweder in eigene „Reservate“ zurückzuziehen oder aber unter entsprechender psychosozialer Begleitung wie jeder andere Bürger Arbeits-, Wohn- oder Freizeitmöglichkeiten in der Kommune wahrzunehmen. Demnach brauchen wir weiterhin sowohl separate als auch integrierte Angebote. 


\section{Literatur}

1 The American Heritage Stedman's Medical Dictionary, $2^{\text {nd }}$ Edition. Boston: Houghton Mifflin Company, 2004

2 Peters HU. Wörterbuch der Psychiatrie, Psychotherapie und medizinischen Psychologie. 3. Aufl. München: Urban \& Schwarzenberg, 1999

3 Bramesfeld A. Wie gemeindenah ist die psychiatrische Versorgung in der Bundesrepublik Deutschland? Psychiat Prax 2003; 30: 256-265

4 Peukert R, Goldbach H (Pro), Vorbach E-U (Kontra). Der Integrierte Behandlungs- und Rehabilitationsplan (IBRP) und die Hilfeplankonferenz (HPK). Psychiat Prax 2006; 33: 3-5
5 Winkler I, Richter-Werling M, Angermeyer MC. Strategien gegen die Stigmatisierung psychisch kranker Menschen und ihre praktische Umsetzung am Beispiel des Irrsinnig Menschlich e.V. Gesundheitswesen 2006; 68: 708-713

6 Finzen A. Psychose und Stigma. Bonn: Psychiatrie-Verlag, 2001

7 Eikelmann B, Richter D, Reker T, Brieger P. Pro und Kontra: Gemeindepsychiatrie in der Krise? Psychiat Prax 2005; 32: 269-271 